\title{
String-wrapped Rotating Disks
}

\author{
Joseph O'Rourke ${ }^{1}$ \\ Smith College, Northampton, MA 01063,USA, \\ orourke@cs.smith.edu, \\ WWW home page: http://cs.smith.edu/ orourke/
}

\begin{abstract}
Let the centers of a finite number of disjoint, closed disks be pinned to the plane, but with each free to rotate about its center. Given an arrangement of such disks with each labeled + or - , we investigate the question of whether they can be all wrapped by a single loop of string so that, when the string is taut and circulates, it rotates by friction all the $\oplus$-disks counterclockwise and all the $\ominus$-disks clockwise, without any string-rubbing conflicts. We show that although this is not always possible, natural disk-separation conditions guarantee a solution. We also characterize the hexagonal "penny-packing" arrangements that are wrappable.
\end{abstract}

\section{Introduction}

Let $\mathcal{A}$ be a collection of $n$ disjoint closed disks in the plane, each labeled + or - , called $\oplus$ - and $\ominus$-disks respectively. We seek to wrap them all in one continuous loop of string so that, were one of the disks rotated by a motor, all the others would spin by friction with the string/belt in a direction consistent with the labeling: counterclockwise (ccw) for $\oplus$-disks and clockwise (cw) for $\ominus$-disks. See Figure 1. We call a wrapping proper if it satisfies these conditions:

1. The string is taut: it follows arcs of disk boundaries and disk-disk bi-tangents only.

2. Each disk boundary circle has a positive-length arc in contact with the string. (It is acceptable for the string to wrap around a disk more than once.)

3. One of the two possible circulation directions (i.e., orientations) for the string loop rotates each disk in the direction consistent with its labeling.

4. If the string contacts a point of a disk boundary circle, its circulation there must be in the direction consistent with that disk's label, i.e., there is no rubbing conflict.

We permit the string to cross itself. Indeed such crossings are necessary: for a pair of $\oplus$ - and $\ominus$ disks, the string must form a crossing figure- 8 shape regardless of their radii and placement. Although the conditions for a proper wrapping are suggested by physical analogy, the pursuit here is not driven by any application. Henceforth a proper wrapping will often be called simply a wrapping.

Proper string wrappings are a variation on the "conveyor-belt" wrappings introduced by Abellanas in 2001 (but not published until [1]), and further studied 


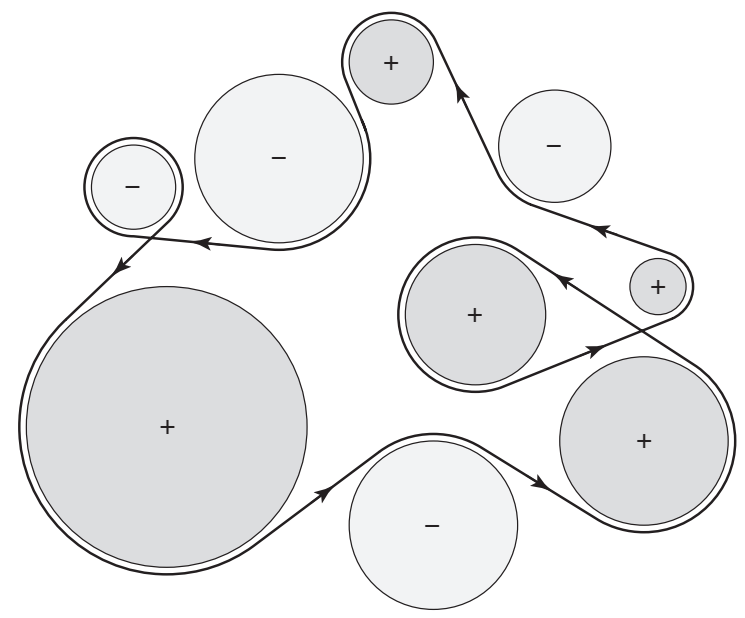

Fig. 1. A proper wrapping of disks with a loop of string: each $\oplus$-disk rotates counterclockwise, each $\ominus$-disk clockwise.

in [2]. The belts in Abellanas' model differ from string wrappings in that the disk rotation directions are not pre-specified, and the belt cannot self-cross. These differences considerably change the character of the problem, although the questions raised are analogous.

We show that not all arrangements of disks have a proper wrapping, but that various separation conditions guarantee proper wrappings. For example, every collection of unit disks has a proper wrapping when each pair is separated by a distance of 0.31 or more. We focus particularly on "penny-packing" arrangements of congruent disks, where we obtain a characterization of the wrappable arrangements. A characterization of wrappability for arbitrary arrangements remains for future work.

\section{Unwrappable arrangements}

An example of an unwrappable arrangement is shown in Figure 2(a). It consists of one unit disk surrounded by six others, arranged in a hexagonal penny-packing pattern, except the disks are just barely disjoint. We now argue that this configuration is unwrappable.

The central disk $A$ must have a positive-length arc of ccw string touching it. Because a taut string can only leave the boundary of a disk along a tangent, the string follows at least the arc between two adjacent tangents. In order for the string to reach another disk, say $C$, and contribute a ccw arc, it must first touch another disk, $B$ in the figure, but now rubbing it in a $\mathrm{cw}$ arc. Thus a rubbing conflict is unavoidable. 

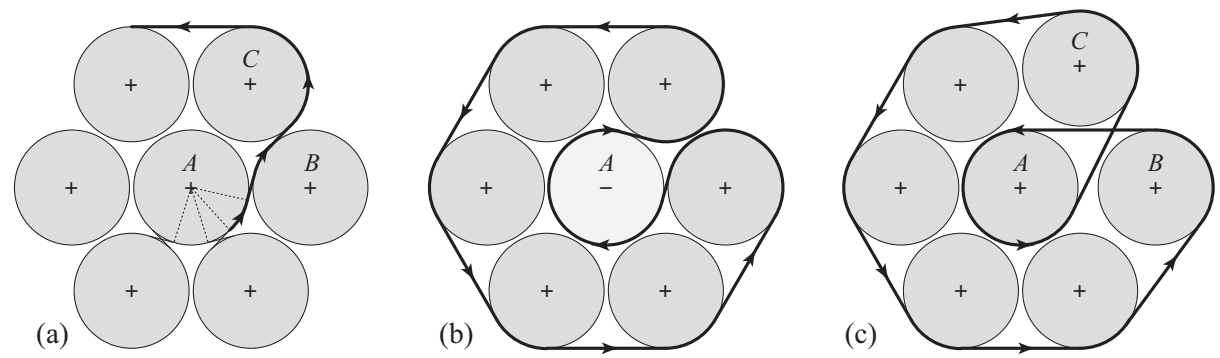

Fig. 2. (a) An unwrappable arrangement of seven unit $\oplus$-disks. (b) Proper wrapping with $A$ an $\ominus$-disk. (c) Proper wrapping with $B$ and $C$ displaced slightly.

Without moving the disks, this arrangement can be properly wrapped with a different pattern of \pm labels. For example, reversing the central disk $A$ enables a proper wrapping: Figure 2(b). Indeed all other \pm patterns of labels (except all - ) in this example are wrappable. Retaining the original + labels but moving two disks slightly also permits the configuration to be wrapped: Figure 2(c).

\section{Separation conditions}

The primary impediment to a proper wrapping is the 4th no-rubbing-conflicts condition. Figure 2(c) indicates that disk-separation conditions may suffice to ensure the existence of a proper wrapping, as separation of the disks separates their tangents and avoids unwanted rubbings. In this section we offer three straightforward conditions that ensure a proper string wrapping exits.

\subsection{Connected Hull-Visibility Graph}

Define two disks to be hull-visible to one another (a symmetric relation) if and only if the (closed) convex hull of the disks does not intersect any other disk; see Figure 3. If two disks can see one another in this sense, then none of their four

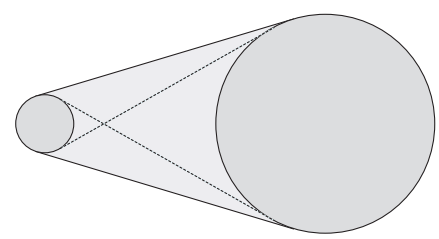

Fig. 3. Two disks are visible to one another if their hull does not intersect any other disk.

bi-tangents are blocked (or even touched) by any other disk. 
For an arrangement $\mathcal{A}$ of disks, define their hull-visibility graph $G_{V}(\mathcal{A})=G_{V}$ to have a node for each disk, and an arc connecting two disk nodes if and only if the disks are hull-visible to one another. Call the hull of a pair of disks connected in $G_{V}$ to the edge corridor for that edge.

Lemma $1\left(G_{V}\right)$. If $G_{V}(\mathcal{A})$ is connected, then there is a proper wrapping of $\mathcal{A}$.

Proof. The proof is by induction, with the hypothesis that the string lies within the union of the edge corridors for all the edges in $G_{V}$. Let $H_{k}$ be a connected component of $G_{V}$ that includes exactly $k$ nodes, and assume the corresponding $k$ disks have been properly wrapped with string loop $S^{\prime}$ by the induction hypothesis. Let $D$ be a disk whose node is not in $H_{k}$, but which is connected by an edge $e=\left(D, D^{\prime}\right)$ to $H_{k}$. Because $e \in G_{V}, D$ can see $D^{\prime}$, and so the edge corridor of $e$ is not blocked by any disk. It is possible that the edge corridor is crossed by segments of $S^{\prime}$ (see Figure 4), but none of those segments can intersect $D$, because by hypothesis $S^{\prime}$ remains within edge corridors, which $D$ cannot intersect.

$S^{\prime}$ must touch $D^{\prime}$ in a positive-length arc, oriented consistent with the \pm label of $D^{\prime}$. Regardless of the position of this arc with respect to $D$, it is possible to wrap the string $S$ around $D^{\prime}$ to reach the relevant bi-tangency points, and then follow those tangents within the corridor of $e$ out to wrap around $D$, regardless of the sign labels of $D^{\prime}$ and $D$. Thus we have incorporated $D$ into $H_{k+1}$, maintaining the hypothesis that the string $S$ lies in the edge-corridor union.

The conditions of this lemma are by no means necessary for the existence of a proper wrapping: $G_{V}$ for the configuration in Figure 2(b) is completely disconnected - seven isolated nodes - and yet it can be properly wrapped.

\subsection{Unit disks halo}

The sufficiency condition of Lemma 1 is a global property of the arrangement $\mathcal{A}$ of disks, not immediately evident upon inspection. Next we explore local separation conditions that allow us to conclude that $G_{V}$ is connected.

Define an $\alpha$-halo, $\alpha>0$, for a disk $D$ of radius $r$ to be a concentric disk $D^{\prime}$ of radius $(1+\alpha)$ such that no other disk of $\mathcal{A}$ intersects $D^{\prime}$.

Lemma 2 (Unit Halo). Let $A, B$, and $C$ be three unit disks with centers at $a, b$, and $c$ respectively, each with $\alpha$-halos for $\alpha=4 / \sqrt{3}-2 \approx 0.31$. Then, if $C$ intersects the $(A, B)$ corridor, $c$ is closer to $a$ and to $b$ than is a to $b:|a c|<|a b|$ and $|b c|<|a b|$.

Proof. Figure 5(a) illustrates the claim of the lemma. The calculation of $\alpha$ follows from the limiting configuration shown in Figure 5(b), where $\triangle a b c$ is equilateral and $(2+\alpha) \frac{\sqrt{3}}{2}=2$. In this configuration, if $c$ is moved left or right along a horizontal, so that it still just intersects the $(A, B)$ corridor, its $\alpha$-halo intersects $A$ or $B$ respectively. 

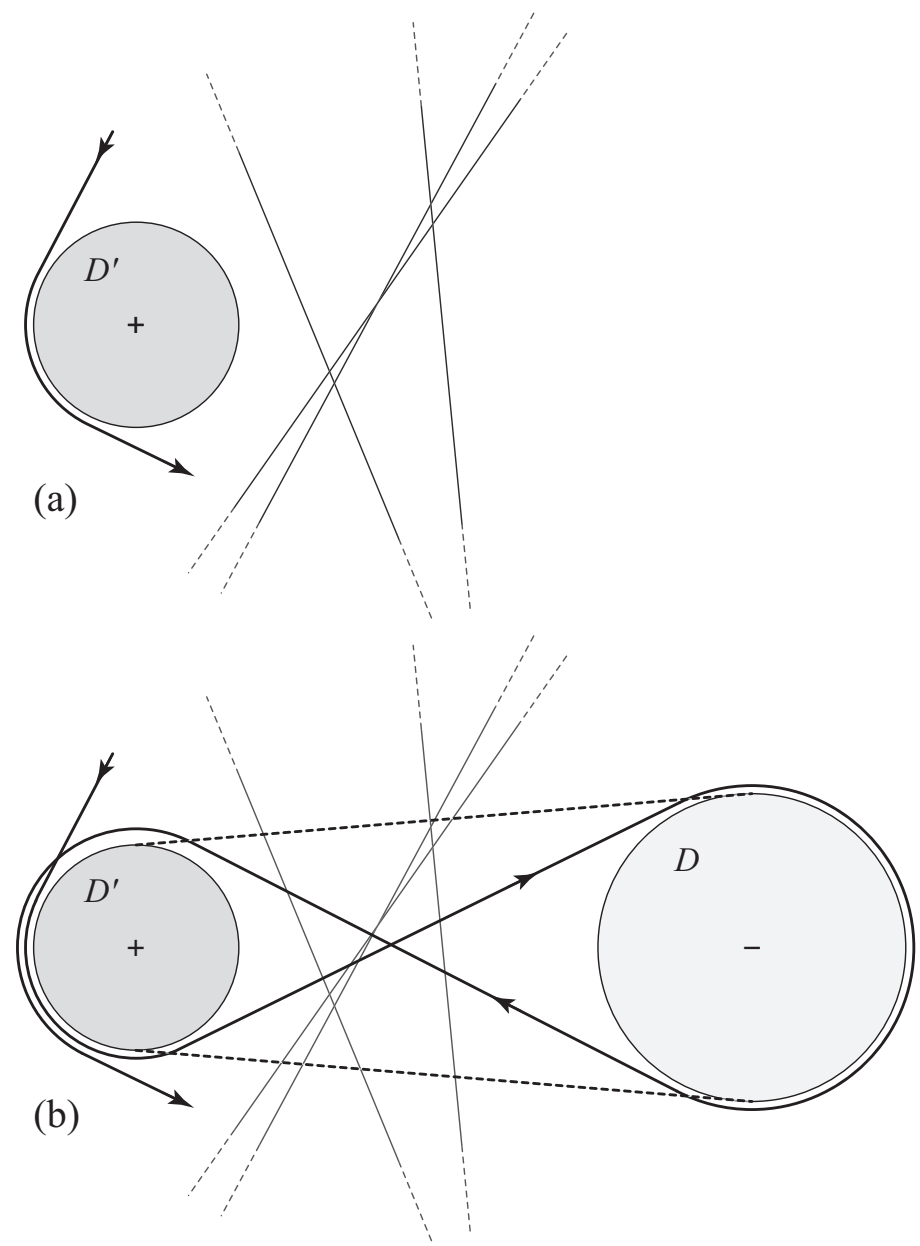

Fig. 4. (a) $D^{\prime}$ is a disk whose node is in $H_{k}$. (b) $D$ can connect to $D^{\prime}$ inside their corridor by wrapping once around $D^{\prime}$. 


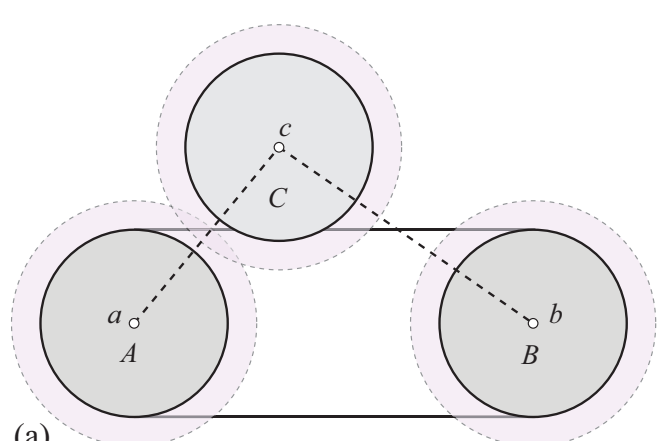

(a)

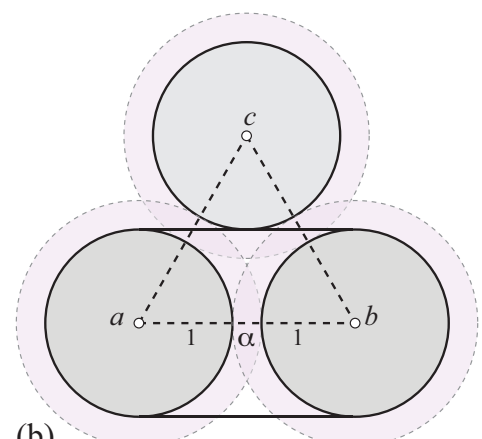

(b)

Fig. 5. (a) Whenever $C$ blocks (intersects) the $(A, B)$ corridor, $c$ is closer to $a$ and $b$ than $|a b|$. (b) The limiting situation.

Theorem 1 (Unit Disks). An arrangement $\mathcal{A}$ of unit disks with $\alpha$-halos, $\alpha=$ $4 / \sqrt{3}-2$, has a connected visibility graph $G_{V}(\mathcal{A})$, and so can be properly wrapped.

Proof. We show that $G_{V}$ is connected by constructing its minimal spanning tree via Prim's algorithm. At any stage in that algorithm, a subset of the nodes have been connected into a tree $T^{\prime}$. Then a shortest edge $e=(a, b)$ is selected such that $a \in T^{\prime}$ and $b \notin T^{\prime}$, and $T^{\prime}$ is updated to $T=T^{\prime} \cup(a, b)$. (This addition of $e$ cannot create a cycle because $b \notin T^{\prime}$.)

In our situation, $e=(A, B)$, with the edge length the distance between the disk centers, $|a b|$. (We will use $A$ and $a$ interchangeably to indicated the nodes of $G_{V}$.) Now we claim that the corridor for $e$ cannot be intersected by any other disk in $\mathcal{A}$. Suppose to the contrary that $C$ obstructs the $(A, B)$ corridor. Then Lemma 2 says that $|a c|<|a b|$ and $|b c|<|a b|$. Consider two cases. First, suppose $c \in T^{\prime}$. Then because $|c b|<|a b|$, we have identified a shorter edge between a node $c$ in $T^{\prime}$ and a node $b$ not in $T^{\prime}$. Second, suppose $c \notin T^{\prime}$. Then because $|a c|<|a b|$, we have again identified a shorter edge between $a \in T^{\prime}$ and $c \notin T^{\prime}$. Both possibilities contradict the choice of $(a, b)$ by the algorithm. Therefore, Prim's algorithm will indeed construct a spanning tree of $G_{V}$. Knowing that $G_{V}$ is connected, we can apply Lemma 1 to conclude that $\mathcal{A}$ can be properly wrapped.

\subsection{Arbitrary radii halo}

For disks of different radii, we define the distance between them to be the distance between their bounding circles (rather than their centers). The assumption in Theorem 1 that all disks are congruent can be removed at the cost of a significant increase in the value of $\alpha$.

Note that, for disks of arbitrary radii, we must allow for a disk radius to be arbitrarily small, effectively a point regardless of $\alpha$. Thus the strategy to avoid 
blockage of a $(A, B)$ corridor is to cover it entirely with the $\alpha$-halos of $A$ and $B$, for no $C$ can penetrate these halos by definition.

Lemma 3 ( $\alpha=1$ Halo). The conclusion of Lemma 2 holds for three disks $A$, $B$, and $C$ of arbitrary radius if $\alpha=1$.

Without loss of generality we may assume that $A$ has a radius $r>1, B$ has unit radius, and $C$ has an arbitrarily small radius. The only proof I have found for this lemma is a brute-force computation of the coverage of the corridor by the $\alpha$-halos of $A$ and $B$, as illustrated in Figure 6 . The calculation shows that

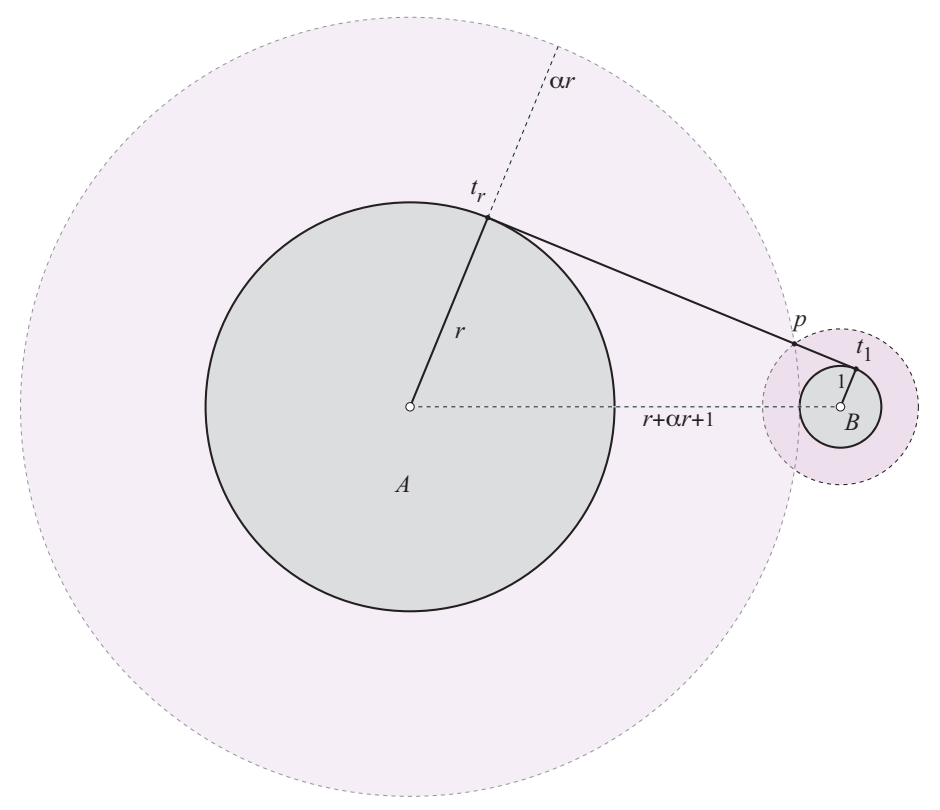

Fig. 6. Covering the corridor for $(A, B)$ by $\alpha$-halos.

$\alpha$ ranges from $2 / 3$ for $r=1$ and approaches but never exceeds 1 as $r \rightarrow \infty$. Because the calculation is tedious and the result of limited interest, we leave it as a claim.

The analog of Theorem 1 follows immediately by an identical proof, only invoking Lemma 3 rather than Lemma 2:

Theorem 2 ( $\alpha=1$ Halo). Any arrangement $\mathcal{A}$ disks with $\alpha$-halos, $\alpha=1$, has a connected visibility graph $G_{V}(\mathcal{A})$, and so can be properly wrapped.

\section{Penny Arrangements}

The visibility and separation conditions in the preceding section guarantee the existence of sufficiently many unblocked bi-tangents to support a proper wrap- 
ping. Ideally we would like precise characterization of the wrappable arrangements. In some sense this is easily obtained: an arrangement is wrappable if and only if a certain graph representing the bi-tangents has a spanning cycle. But this is unsatisfying: we would prefer a characterization in terms of more local, or at least easily recognizable, characteristics of the arrangement. I have only achieved this for the penny-packing arrangements epitomized by Figure 2(a,b), as described in this section. In some sense these arrangements are the obverse of well-separated arrangements, for nearly all bi-tangents are blocked.

\subsection{Characterization Theorem}

Let $H$ be the hexagonal lattice, also known as the equilateral triangle lattice (wallpaper group p6m), with each edge of each triangle of unit length. We can think of $H$ as an infinite (plane) graph.

Disks of radius $\frac{1}{2}-\varepsilon$ are centered on some finite subset of the points of $H$, where $\varepsilon>0$ is small. Any $\varepsilon<\frac{1}{2}-\sqrt{3} / 4 \approx 0.07$ suffices to ensure that the only tangents between adjacent disks are the "cross" X-tangents; see Figure 7. Each

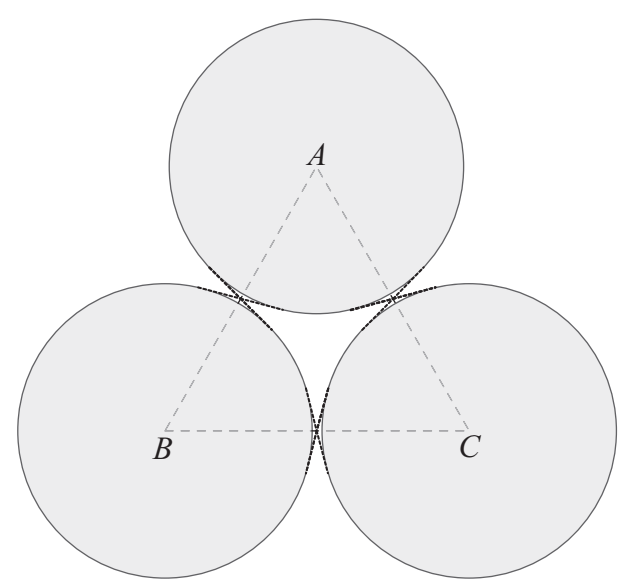

Fig. 7. For a unit equilateral underlying lattice, the disk radii are nearly $\frac{1}{2}$.

disk has up to six neighbors following the edges of $H$.

We will define four subgraphs of $H$, three to describe the characterization theorem and one more used in the proof: $G, G^{+}, G^{-}$, and $G_{T}$.

Define the plane graph $G \subset H$ to have a node for each disk, and an arc for each pair of disks that are neighbors in $H$. See Figures 8 and 9.

We restrict attention to a subclass of all possible arrangements of disks on $H$. Define a penny arrangement of disks to be one whose disk radii are close to $\frac{1}{2}$ in the sense discussed previously, and which satisfies two additional assumptions. Let $\partial G$ be the outer face of $G$. 


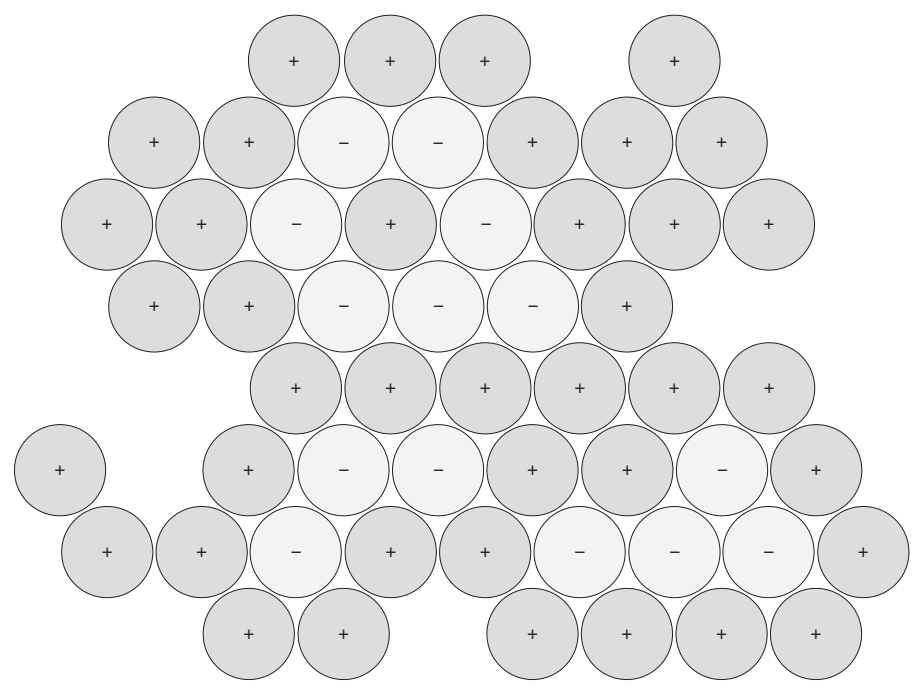

Fig. 8. A penny arrangement on a hexagonal lattice $H$.

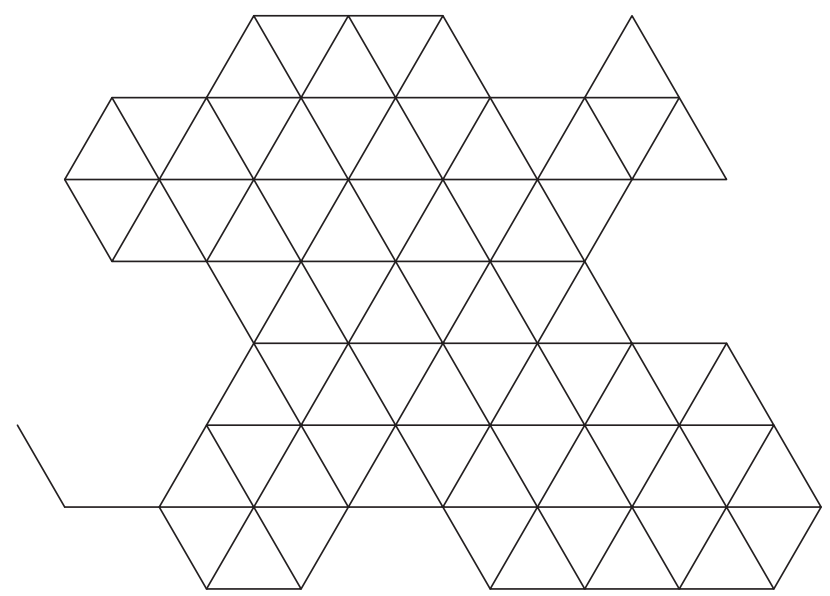

Fig. 9. The graph $G \subset H$ recording all disk adjacencies. 
1. $G$ is connected and has no holes, in the sense that every lattice point inside $\partial G$ is a node of $G$.

2. Every node of $\partial G$ is an $\oplus$-disk.

The example in Figure 8 satisfies these assumptions. I believe these assumptions are not essential, but they allow a more transparent proof. Relaxing these assumptions will be discussed at the end of Section 4.2.

Now we define $G^{+}$and $G^{-} . G^{+}$is the subgraph of $G$ restricted to nodes corresponding to $\oplus$-disks, and similarly $G^{-}$is the subgraph restricted to $\ominus$-disks. In general both $G^{+}$and $G^{-}$consist of several components. Each component of $G^{+}$is adjacent to a component of $G^{-}$via a least one edge of $G$ (because $G$ is connected). See Figure 10.

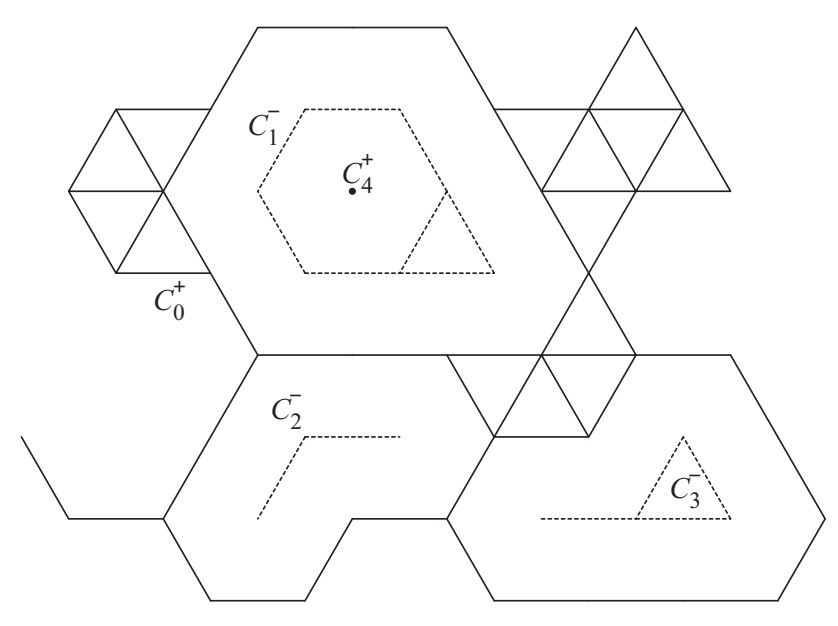

Fig. 10. $G^{+}$and $G^{-}$for the arrangement in Figure 8. $G^{+}$consists of two components, $C_{0}^{+}$and $C_{4}^{+}$, and $G^{-}$consists of three components, $C_{1}^{-}, C_{2}^{-}$, and $C_{3}^{-}$.

We now introduce notation that will permit us to characterize the wrappable penny arrangements in terms of structural constraints on $G^{+}$and $G^{-}$. We phrase these definitions in terms of $G^{+}$but the same hold for $G^{-}$.

A hole in a component $C^{+}$of $G^{+}$is a face that contains a lattice point not occupied by a $\oplus$-disk. ( $C_{0}^{+}$in Figure 10 has three holes; $C_{1}^{-}$has one hole.) A gate edge of a hole in $C^{+}$is one whose removal joins the hole with another hole of $C^{+}$, or with the exterior of $C^{+}$. A hole of $C^{+}$is shallow if it can be connected to the exterior of $C^{+}$by removal of a sequence of gate edges. Figure 11(b) shows an example.

Call the graph shown in Figure 11(a) the hexagon graph; it coincides with $G^{+}$for Figure 2(a). The characterization is in terms of these properties: 


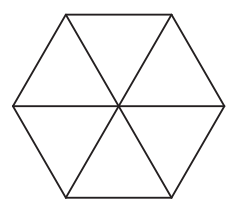

(a)

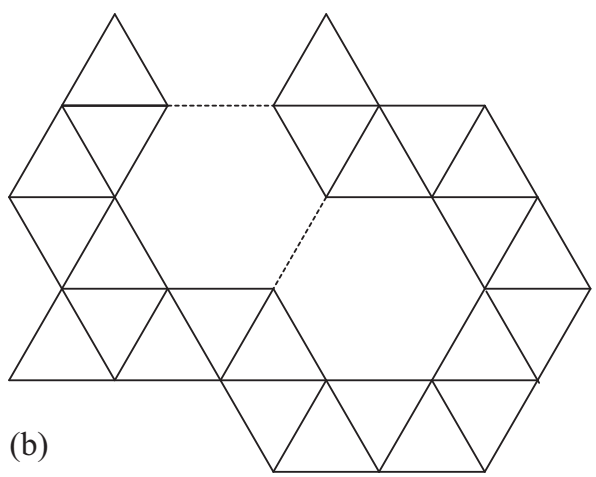

Fig. 11. (a) The hexagon graph. (b) A component (of $G^{+}$or $G^{-}$) whose holes are shallow. The two gate edges are shown dashed.

Property (1): No component of $G^{+}$or $G^{-}$contains the hexagon graph as a subgraph.

Property (2): All holes of every component of $G^{+}$and $G^{-}$are shallow.

Theorem 3 (Penny Arrangements). A penny arrangement $\mathcal{A}$ of disks is wrappable if and only if $G^{+}$and $G^{-}$satisfy Properties (1) and (2).

\subsection{Proof of Theorem 3}

Although I find it natural to see Theorem 3 by wrapping each component of $G^{+}$and $G^{-}$separately, and using the two properties to join the wrappings, this seems to lead to an awkward proof. So here we take a different approach using a tangency graph, which leads to a less natural wrapping, but a simpler a proof.

The key observation is that the string can not wrap directly between two adjacent disks of the same label, unless they are on $\partial G$. The reason is that the desired tangent touches a third disk, unless that third disk is not present because it would be outside of $G$. Examining Figure 7 again shows that only two oppositely labeled adjacent (internal) disks can directly wrapped one to the other. This suggests defining a directed tangent graph $G_{T}$ to include for each edge of $G$ connecting a $\oplus$-disk to an $\ominus$-disk, an edge in both directions, and, for each edge of $\partial G$, the ccw-directed edge between the connected $\oplus$-disks. (Recall our assumption that the nodes of $\partial G$ are all $\oplus$-disks.) See Figure 12. $G_{T}$ simply records all the bi-tangents that might be followed disk-to-disk. Note that all the edges of $G_{T}$ that are not connecting two nodes of $\partial G$ are bidirectional, connecting $\oplus$ - and $\ominus$-disks, and that the only edges of $G_{T}$ connecting like-labeled disks are those on $\partial G$.

A directed spanning cycle in a directed graph is a collection of oriented arcs that touch each node of the graph and form a cycle. Both nodes and arcs may be visited several times by the cycle. Here is the nearly obvious characterization mentioned previously. 


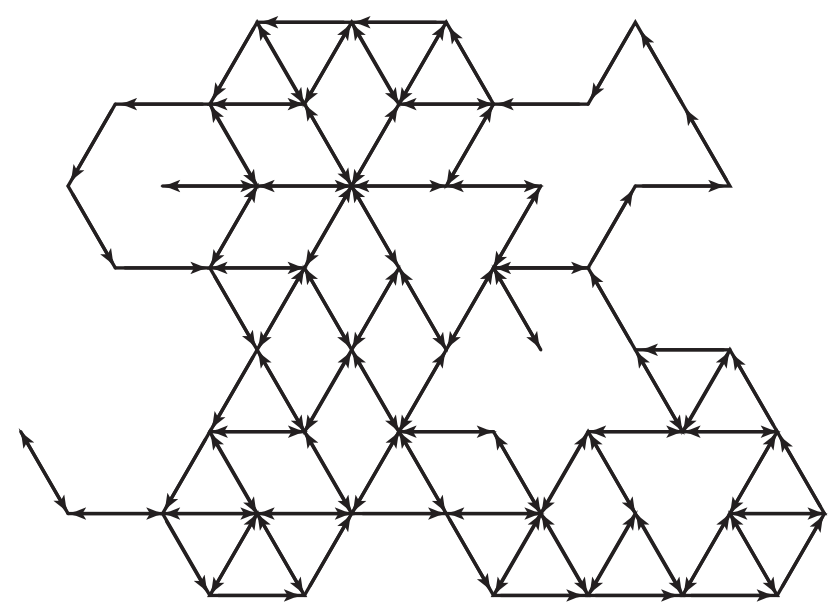

Fig. 12. $G_{T}^{+}$for the arrangement in Figure 8. $\partial G$ includes a counterclockwise cycle.

Lemma $4\left(G_{T}\right)$. A penny arrangement is wrappable if and only if $G_{T}$ contains a directed spanning cycle.

Proof. Suppose $G_{T}$ contains a directed spanning cycle $\left(\overrightarrow{e_{1}}, \overrightarrow{e_{2}}, \ldots, \overrightarrow{e_{m}}\right)$. We incrementally construct a string that turns each disk. Assume we have a string $S_{k-1}$ that corresponds to $\left(\overrightarrow{e_{1}}, \ldots, \overrightarrow{e_{k-1}}\right)$; we seek to extend it to include $\overrightarrow{e_{k}}=(A, B)$, where $A$ and $B$ are the two disks connected by the tangent corresponding to $\overrightarrow{e_{k}}$. First, wrap the string around $A$ consistent with its label so that it reaches the tangent departure point. Then follow the tangent from $A$ to $B$. By definition of $G_{T}$, this necessarily works, in that the tangent is consistent with the \pm labels of the disks and does not rub against any other disk. See Figure 13(a,b). The new string $S_{k}$ is now poised for the next extension. This establishes the "if"-direction of the lemma claim.

For the "only if"-direction, suppose that there is a proper wrapping of the arrangement by a string $S$. Then it is straightforward to map $S$ to the tangents it follows, and so to a corresponding spanning cycle in $G_{T}$.

Now we connect the cycle in $G_{T}$ to the structural properties of $\mathcal{A}$.

Lemma 5 (Props.1\&2). $G_{T}$ contains a directed spanning cycle for a penny arrangement $\mathcal{A}$ if and only if $\mathcal{A}$ satisfies Properties (1) and (2),

Proof. Assume first that $\mathcal{A}$ satisfies the two properties. By the definition of a penny arrangement, $G_{T}$ has a counterclockwise cycle including every node of $\partial G$. By Property (2) applied to the outermost component of $G^{+}$, there must be at least one edge $\vec{e}=(A, B)$ of this cycle representing a gate edge, and so whose endpoints are connected to a $\ominus$-disk $D$. See Figure 13(c,d). 

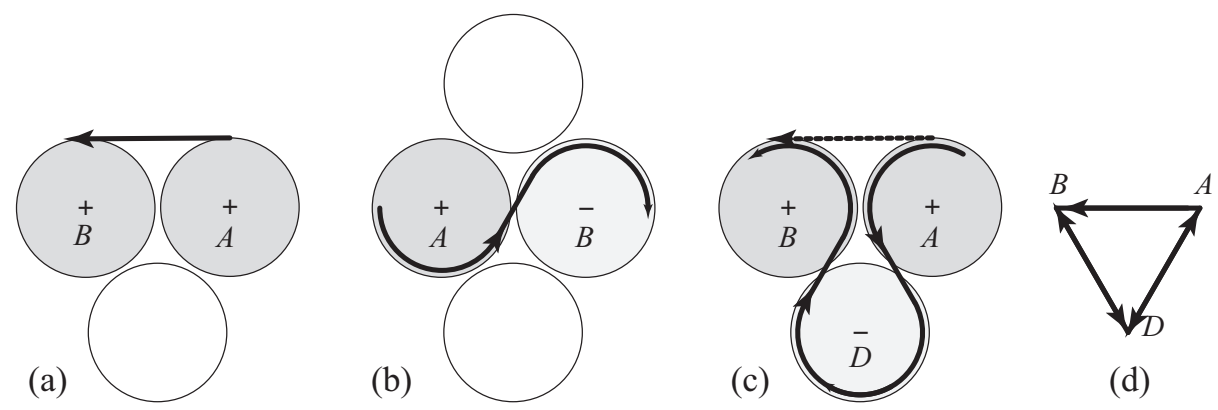

(d)

Fig. 13. A string connecting two disks adjacent via a directed arc of $G_{T}$ : (a) $(A, B) \subseteq$ $\partial G$. (b) The tangent does not touch any disk other than $A$ and $B$. (c) Here $(A, B)$ is a gate edge guaranteed by Property (2), and so edges of $G_{T}$ connect to an adjacent disk $D$, as in (d).

We now argue that the bidirectional arcs of $G_{T}$ connect all the disks in $G$. Property (1) shows that every disk is connected to another: forbidding the hexagon subgraph means that no $\oplus$-disk can be entirely surrounded by $\oplus$-disks. Property (2) guarantees that every component of $G^{+}$or $G^{-}$is connected in $G_{T}$ to its exterior via a series gate edges. A simple induction on the depth of nesting of one component inside another leads to the connected conclusion. Start with a deepest component (e.g., $C_{4}^{+}$in Figure 10). All of its disks are connected in $G_{T}$ to the surrounding component $\left(C_{1}^{-}\right.$in the figure). The series of gate edges that connect the holes to the exterior of the component guarantee a connection up the nesting hierarchy.

Now take a spanning tree $T$ of $G_{T}$, following bidirectional edges (i.e., not employing the counterclockwise edges of $G_{T}$ corresponding to $\partial G$ ). Traversing $T$, and connecting through $D$ to the cycle for $\partial G$ (cf. Figure 13(c)), results in a proper wrapping of the arrangement. This establishes the "if"-direction of the lemma.

For the "only-if" direction, assume Properties (1) and (2) fail to hold for $\mathcal{A}$. Certainly if a component includes a hexagon subgraph (violating Property (1)), there can be no spanning cycle, because the central disk has no incident edges in $G_{T}$. So assume Property (2) fails to hold for some component $C^{+}$. This means that removal of all gate edges for its holes leaves a merged hole $C^{-}$that still cannot connect to the exterior of $C^{+}$. This can only happen if $C^{-}$is surrounded by a "padding" of $\oplus$-disks thick enough so that no gate edge connects to the exterior. The simplest example is illustrated in Figure 14. In this case it is easy to see that, although the $\ominus$-disks in $C^{-}$connect by edges of $G_{T}$ to $C^{+}$, the padding isolates these connections from the exterior of $C^{+}$, disconnecting $G_{T}$. Thus, again $G_{T}$ cannot contain a spanning cycle.

Lemmas 4 and 5 together establish Theorem 3.

I believe the assumption in Theorem 3 that $\partial G$ is composed entirely of $\oplus$ disks can be removed by surrounding a given arrangement with "virtual" $\oplus$-disks, 


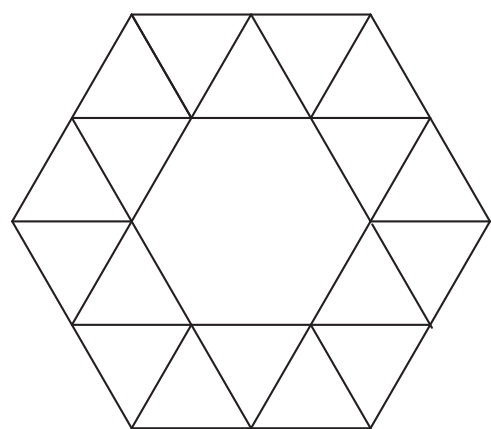

Fig. 14. Property (2) fails: $C^{+}$contains a hole without a gate edge. Here only edges of $G^{+}$are shown. None of the diagonals connecting the inner to the outer hexagon can be edges of $G_{T}$, because they connect $\oplus$-disks not both on $\partial G$.

and later removing them. There also seems no impediment permitting $G$ to have holes, or to be disconnected. But these extensions perhaps best await a proof technique that encompasses them without effort.

\section{Discussion}

It is natural to hope that some analog of Theorem 3 holds for arbitrary arrangements. However, I have not found a formulation that avoids devolving to a version of Lemma 4, relying on the existence of a cycle in a tangency graph analogous to $G_{T}$. A clean characterization remains open.

A second open question is to find a shortest wrapping when proper wrappings exist. For widely spaced disks, the analogy with Euclidean TSP suggests this may be NP-hard, but the situation is less clear for congested arrangements.

Acknowledgments. I thank Manuel Abellanas for graciously forgiving my oversight of his priority, and I thank Erik Demaine for the observation connecting to the ETSP. This paper benefited from insightful comments from referees and attendees at the XIV Spanish Meeting on Computational Geometry.

\section{References}

1. Abellanas, M.: Conectando puntos: poligonizaciones y otros problemas relacionados. Gaceta de la Real Sociedad Matematica Española 11(3) (2008) 543-558

2. Demaine, E.D., Demaine, M.L., Palop, B.: Conveyer-belt alphabet. In Aardse, H., van Baalen, A., eds.: Findings in Elasticity. Pars Foundation, Lars Müller Publishers (April 2010) 86-89 